\title{
Polyglycerolsulfate Functionalized Gold Nanorods as Optoacoustic Signal Nanoamplifiers for In Vivo Bioimaging of Rheumatoid Arthritis
}

\author{
Jonathan Vonnemann ${ }^{1 凶}$, Nicolas Beziere ${ }^{2 凶}$, Christoph Böttcher $^{3}$, Sebastian B. Riese ${ }^{4}$,Christian Kuehne ${ }^{4}$ \\ Jens Dernedde ${ }^{4}$ Kai Licha5, Claudio von Schacky6, Yvonne Kosanke6, Melanie Kimm6, Reinhard Meier6, \\ Vasilis Ntziachristos ${ }^{2}$, Rainer Haag ${ }^{1}$ \\ 1. Institute of Chemistry and Biochemistry, Freie Universität Berlin, 14195 Berlin, Germany; \\ 2. Chair for Biological Imaging, Technische Universität München, 81675 Munich, Germany and Institute for Biological and Medical Imaging, \\ Helmholtz Zentrum München, 85764 Neuherberg, Germany; \\ 3. Research Centre of Electron Microscopy and Core Facility BioSupraMol, Freie Universität Berlin, 14195 Berlin, Germany; \\ 4. Institute of Laboratory Medicine, Clinical Chemistry and Pathobiochemistry, Charité-Universitätsmedizin Berlin, 13353 Berlin, Germany; \\ 5. mivenion $\mathrm{GmbH}, 10115$ Berlin, Germany; \\ 6. Department of Radiology, Klinikum rechts der Isar, Technische Universität München, 81675 Munich, Germany.
}

$\triangle$ Corresponding authors: Jonathan Vonnemann, jonathan.vonnemann@chemie.fu-berlin.de; Nicolas Beziere, nicolas.beziere@helmholtz-muenchen.de.

() Ivyspring International Publisher. This is an open-access article distributed under the terms of the Creative Commons License (http://creativecommons.org/ licenses/by-nc-nd/3.0/). Reproduction is permitted for personal, noncommercial use, provided that the article is in whole, unmodified, and properly cited.

Received: 2014.01.07; Accepted: 2014.02.02; Published: 2014.03.20

\begin{abstract}
We have synthesized a targeted imaging agent for rheumatoid arthritis based on polysulfated gold nanorods. The CTAB layer on gold nanorods was first replaced with PEG-thiol and then with dendritic polyglycerolsulfate at elevated temperature, which resulted in significantly reduced cytotoxicity compared to polyanionic gold nanorods functionalized by non-covalent approaches. In addition to classical characterization methods, we have established a facile UV-VIS based $\mathrm{BaCl}_{2}$ agglomeration assay to confirm a quantitative removal of unbound ligand. With the help of a competitive surface plasmon resonance-based L-selectin binding assay and a leukocyte adhesion-based flow cell assay, we have demonstrated the high inflammation targeting potential of the synthesized gold nanorods in vitro. In combination with the surface plasmon resonance band of AuNRs at $780 \mathrm{~nm}$, these findings permitted the imaging of inflammation in an in vivo mouse model for rheumatoid arthritis with high contrast using multispectral optoacoustic tomography. The study offers a robust method for otherwise difficult to obtain covalently functionalized polyanionic gold nanorods, which are suitable for biological applications as well as a low-cost, actively targeted, and high contrast imaging agent for the diagnosis of rheumatoid arthritis. This paves the way for further research in other inflammation associated pathologies, in particular, when photothermal therapy can be applied.
\end{abstract}

Key words: gold nanorods, optoacoustic, dendritic polyglycerolsulfate, inflammation, polyanion, MSOT.

\section{Introduction}

The use of multiple wavelengths for illumination, advanced data processing methods, and identification and distribution of photoabsorbers based on their light absorbance spectra enables multispectral optoacoustic tomography (MSOT) to exceed the depth and resolution of classical optical imaging methods [1]. As pulsed laser light reaches photoabsorbers, pressure waves are emitted as a result of their ther- 
moelastic expansion, which can be detected by classical ultrasonic transducers [2]. While classical optical methods require challenging multi-step syntheses of organic dyes, MSOT can circumvent this problem because of its ability to image photo-absorbance in vivo without relying on fluorescence. Anisotropic gold colloids, i.e., gold nanorods (AuNRs), are extremely well suited as a contrast agent because of their predominantly non-radiative de-excitation which results in one of the highest photothermal conversions and thus efficiency known in literature [1,3]. Consequently, they are well detectable by MSOT as recently shown in different models [4,5]. Their anisotropy results in a transversal and longitudinal surface plasmon resonance (TSPR/LSPR) band, of which the latter is especially interesting for in vivo applications due to its adjustable absorption wavelength that is dependent on the dimension of the nanorod [6,7]. An LSPR band that absorbs within the "water window" absorption range of 700-1200 nm, therefore minimizing photon absorption by tissue components, is thus readily accessible just by increasing the aspect ratio of the AuNRs [8]. Nevertheless, capitalizing on the favorable optoacoustic properties in imaging of inflammation via MSOT requires targeted AuNRs. Moieties for the targeting of inflammation associated pathologies, i.e., in cancer, mostly consist of expensive peptides or synthetically challenging oligosaccharides. Low-cost polymeric dPGS, however, has been shown by our group to exhibit high affinity towards leukocytes and inflamed endothelia, mainly governed by their interaction with L- and P-selectin $[9,10]$. Selectins and their ligands play prominent roles in inflammation and disease and are crucial for the innate immune response [11]. Effective leukocyte recruitment requires these cell adhesion molecules, as demonstrated in mice that lack selectins or selectin ligands [12,13]. P- and E-selectin are expressed on vascular epithelia at high density at sites of inflammation. Therefore it is not surprising that both molecules have been used as targets for drug delivery and imaging purposes $[14,15]$. Due to the low affinity of the most abundant physiological ligand for all selectins, the tetrasaccharide SiaLe ${ }^{x}$, antibodies were mainly used as high affinity probes for selectin targeting in vivo. Recently an antibody directed fluorescence imaging of E-selectin was successfully applied in a mouse model for arthritis and the authors claimed that the quantified fluorescence signal can be related to a disease score [16]. Besides the interaction of selectins with specific antibodies, numerous experiments confirm an essential electrostatic contribution in L- and P-selectin binding [17-20]. Our group has shown that sulfates are by far the strongest binders for L- and P-selectin of all anions, which explains the high binding affinity of dPGS [21]. The targeting potential of dPGS has already been successfully exploited by our group in several mouse models for the in vivo imaging of inflammation with synthetically challenging organic fluorophores [22-24], while difficulties in the anionic functionalization prohibited the use of readily available gold nanorods as imaging modality. Although gold nanorods can be synthesized by a single-step, gram-scale procedure [25], the functionalization of gold nanorods for biocompatibility and active targeting is not trivial. In order to achieve an anisotropic growth of gold nanorods, a highly concentrated solution of cetyltrimethylammonium bromide $(\mathrm{CTAB})$ is required for the stabilization of the dispersion [8]. This surfactant is not only inherently cytotoxic, it also renders the nanorods cationic in surface charge [8]. The polyanionic functionalization of the gold nanorods is thus challenging, as the strong interaction of anionic substances with the positively charged CTAB prohibits ligand exchange on the gold nanorods. Therefore, several synthetic methods have been established for the anionic functionalization of AuNRs including layer-by-layer (LBL) coating with polyelectrolytes [26,27], silica-functionalization with subsequent grafting from/to [28], and a round-trip phase transfer of the gold nanorods [29]. Layer-by-layer functionalization proved to not be applicable for in vivo applications because the supramolecular structures were unstable under high salt concentrations. The alternative, namely, functionalizing silica coated AuNRs, is chemically demanding as this requires fine-tuned reaction conditions and solvent mixtures for retaining the colloidal stability. We present here, for the first time, a reliable method for the preparation of covalently functionalized polysulfated AuNRs stable under physiological conditions via a thermally induced ligand replacement reaction of PEG. Even though displacement of monothiol ligands for quantification by dithiothreitol (DTT) at room temperature is reported in the literature [30], a thermally induced replacement of PEG-thiol by disulfide modified ligands has not been reported as a functionalization mechanism. As the CTAB and PEG replacement reactions are performed in aqueous solution and do not require additional solvents, this offers a new universal method for the functionalization of gold nanorods with anionic ligands. The intermediate PEGylation step renders the AuNR surface neutral and at the same time sterically stabilizes the colloid. Given the targeting properties of dPGS, they are expected to specifically address inflamed tissue and bind to L-selectin of invading leukocytes as well as to P-selectin expressed on vascular epithilia [22]. Therefore, we employed in this study a well-established murine rheumatoid arthritis model to observe the 
accumulation of AuNR-dPGS at the inflamed region of the joints [31]. For the first time, we were able to show the successful application of an inflammation targeted, colloidal contrast agent for MSOT. The easy functionalization of readily available AuNR-CTAB with the low-costs of dPGS highlights the advantage of nanoparticular imaging agents over conventional organic dyes and establishes a basis for a cheap and efficient diagnosis and photothermal therapy of additional inflammatory diseases. Furthermore, the established thermally induced ligand exchange of PEG offers a novel method for the functionalization of anisotropic gold colloids with hydrophilic ligands.

\section{Experimental}

\section{I Materials}

Air and moisture sensitive reactions were carried out in flame-dried glassware under argon atmosphere. Anhydrous solvents were either commercially purchased from Acros Organics ${ }^{\mathrm{TM}}$ in septum sealed bottles or chemically dried using a MBRAUN SPS 800 solvent purification system. All other chemicals were purchased from Aldrich ${ }^{\mathrm{TM}}$. Cellulose acetate filters $(0.2 \mu \mathrm{m})$ were purchased from Whatman ${ }^{\mathrm{TM}}$. TA-NHS ester was synthesized according to the method described by Bawendi et al. $10 \%$ amine and $85 \%$ sulfate functionalized dendritic polyglycerolsulfate with an average molecular weight of $10 \mathrm{kDa}$ was provided by mivenion $\mathrm{GmbH}$ [32]. Centrifugation was performed with a Hettich ${ }^{\mathrm{TM}}$ Rotina 380R. ${ }^{1} \mathrm{H}-\mathrm{NMR}$ and ${ }^{13} \mathrm{C}-\mathrm{NMR}$ spectra were recorded on a Bruker ${ }^{\mathrm{TM}}$ Biospin spectrometer operating at $700 \mathrm{MHz}$. The chemical shifts are reported in $\delta(\mathrm{ppm})$ and were referenced to the solvent peak. Multiplicities are indicated by s (singlet), d (doublet), $\mathrm{t}$ (triplet), $\mathrm{q}$ (quartet) and $\mathrm{m}$ (multiplet). The corresponding coupling constants J are reported in Hertz $(\mathrm{Hz})$. Data analysis was performed using MestReNova ${ }^{\mathrm{TM}}$ version: 6.0.2. UV/Vis absorption spectra were recorded using a UV/Vis spectrophotometer by Scinco ${ }^{\mathrm{TM}}$ Co., LTD. Data analysis was performed by the corresponding LabProPlus ${ }^{\mathrm{TM}}$ software. Dynamic light scattering and zeta potential measurements were performed on a Zetasizer Nano $\mathrm{ZS}$ analyzer ${ }^{\mathrm{TM}}$ with integrated $4 \mathrm{~mW}$ He-Ne laser, $\lambda=$ $633 \mathrm{~nm}$ (Malvern Instruments ${ }^{\mathrm{TM}}$ Ltd, U.K.). Transmission electron microscopy was conducted on a CM-12 by FEI $^{\mathrm{TM}}$ with an accelerating voltage of $100 \mathrm{kV}$. Graphical analysis of TEM images were performed using JImage v1.43.

\subsection{Chemical Synthesis}

\subsection{Synthesis of TA-dPGS $10 \mathrm{kDa}$}

dPGS-Amine $10 \mathrm{kDa}(500 \mathrm{mg}, 0.05 \mathrm{mmol}$ ) was dissolved in a mixture of dimethylformamide $(16 \mathrm{~mL})$ and distilled water $(4 \mathrm{~mL})$. TA-NHS ester $(15.15 \mathrm{mg}$, $0.05 \mathrm{mmol})$ and $N, N$-diisopropylethylamine $(0.017 \mathrm{~mL}, 0.1 \mathrm{mmol})$ were added and the solution was stirred for $3 \mathrm{~d}$. The solvent was removed in vacuo and the crude product dialyzed against $100 \mathrm{mM}$ sodium chloride solution and distilled water in a regenerated cellulose dialysis tube with a MW cut-off of $2000 \mathrm{~g} / \mathrm{mol}$. After lyophilization, the product was obtained as white crystals (491 mg, 98\%).

${ }^{1} \mathrm{H}-\mathrm{NMR}\left(700 \mathrm{MHz}, \mathrm{D}_{2} \mathrm{O}\right): \delta$ (ppm) 4.90-3.21 (m, 338 H, PG-backbone, S-S- $\left.\mathrm{CH}_{2}-\right) 3.21-3.06(\mathrm{~m}, 2 \mathrm{H}$, S-S-CH $\mathbf{C H}_{2-}$, S-S-CH-), 2.69 (m, 1 H, S- $\left.\mathrm{CH}_{2}-\mathbf{C H}_{2}-\right), 2.48$ $\left(\mathrm{t}, 2 \mathrm{H}, J=7.5 \mathrm{~Hz}, \mathrm{CH}_{2} \mathrm{CO}-\right), 2.20(\mathrm{~m}, 1 \mathrm{H}$, $\left.\mathrm{S}-\mathrm{CH}_{2}-\mathrm{CH}_{2}-\right), \quad 1.95-1.53(\mathrm{~m}, 6 \mathrm{H}$, $\left.\mathbf{C H}_{2}-\mathbf{C H}_{2}-\mathbf{C H}_{2}-\mathrm{CH}_{2} \mathrm{CO}-\right), 1.55$ (t, $2 \mathrm{H}, \mathbf{C H}_{2}$ - initiator) $0.78\left(\mathrm{~m}, 3 \mathrm{H}, \mathbf{C H}_{3}\right.$ - initiator).

${ }^{13} \mathrm{C}-\mathrm{NMR}\left(700 \mathrm{MHz}, \mathrm{D}_{2} \mathrm{O}\right): \delta(\mathrm{ppm}) 177.4(1 \mathrm{C}$, -CO-NH-), 78-66 (PG-backbone), 56.6 (1 C, -S-CH-), 54.5 (1 C, -NH-CH-), 42.7 (1 C, -C- starter), 41.2 (1 C, $\left.-\mathrm{S}-\mathrm{CH}_{2}-\right), \quad 38.3 \quad\left(1 \quad \mathrm{C}, \quad-\mathrm{CH}_{2}-\mathrm{CO}-\right), \quad 35.7 \quad(1 \mathrm{C}$, $\left.-\mathrm{S}-\mathrm{CH}_{2}-\mathrm{CH}_{2}-\right), 33.9$ (1 C, -S-CH-CH $\left.{ }_{2}^{-}\right), 28.0$ (1 C, $-\mathrm{CO}-\mathrm{CH}_{2}-\mathrm{CH}_{2}-\mathrm{CH}_{2}-$ ), 25.1 (1 C, - $\mathrm{CO}-\mathrm{CH}_{2}-\mathrm{CH}_{2}-\mathrm{CH}_{2-}$ ), 22.0 ( $1 \mathrm{C},-\mathrm{CH}_{2}-\mathrm{CH}_{3}$ initiator), $7.0\left(1 \mathrm{C},-\mathrm{CH}_{2}-\mathrm{CH}_{3}\right.$ initiator) sulfur content from elemental analysis: $15.5 \%$

\subsubsection{Synthesis of AuNR-CTAB}

Gold nanorods were synthesized according to a slightly modified procedure described by Nikhil R. Jana [25]. Cetyltrimethylammonium bromide $(18.225 \mathrm{~g}, 50 \mathrm{mmol})$ was added to a solution of hydrogen tetrachloroaureate(III) hydrate $(98.5 \mathrm{mg}$, $0.25 \mathrm{mmol})$ in ultrapure water $(255 \mathrm{~mL})$ at $35^{\circ} \mathrm{C}$ under magnetic stirring. The color changed from yellow to deep red. The solution was cooled down to RT and silver nitrate $(8.48 \mathrm{mg}, 50 \mu \mathrm{mol})$ and ascorbic acid ( $88 \mathrm{mg}, 0.5 \mathrm{mmol}$ ) was added to the reaction mixture, resulting in a colorless solution. After the fast addition of an aqueous solution of sodium borohydride $(25 \mu \mathrm{L}$, $1 \mathrm{mM}$ ) under rapid stirring, the reaction mixture turned from colorless to dark purple within $30 \mathrm{~min}$. The colloidal dispersion was purified twice by centrifugation at $6,500 \mathrm{rpm}$ and $35^{\circ} \mathrm{C}$ for $2 \mathrm{~h}$ and redispersion in water, and was stored in the dark at $4{ }^{\circ} \mathrm{C}$.

\subsubsection{Synthesis of AuNR-PEG}

Poly(ethylene glycol)monomethyl ether thiol (134 mg, $134 \mathrm{mmol}, 1.24 \cdot 10^{5}$ eq.) was added to a dispersion of AuNR-CTAB $(4 \mathrm{~mL}, 270 \mathrm{nM})$ and stirred for $24 \mathrm{~h}$ at RT. The colloidal dispersion was purified twice by centrifugation at $6,500 \mathrm{rpm}$ for $1.5 \mathrm{~h}$ and redispersion in ultrapure water and stored in the dark at $4{ }^{\circ} \mathrm{C}$.

\subsubsection{Synthesis of AuNR-dPGS}

TA-dPGS $10 \mathrm{kDa}\left(270 \mathrm{mg}, 27\right.$ rmol, $1.24 \cdot 10^{5}$ eq.) were added to an aqueous dispersion of AuNR-PEG 
$(15 \mathrm{~mL}, 14.5 \mathrm{nM})$, decanted into $1.5 \mathrm{ml}$ Eppendorf tubes and agitated at $60^{\circ} \mathrm{C}$ for $12 \mathrm{~h}$. The colloidal dispersion was purified five times by centrifugation at $11,000 \mathrm{rpm}$ for $15 \mathrm{~min}$ and redispersion in ultrapure water and stored in the dark at $4{ }^{\circ} \mathrm{C}$.

\subsection{Characterization and biological evaluation}

\subsection{Quantification of dPGS mass-fraction on AuNRs via ATR-FTIR}

The calibration curve was established by measuring the absorption of the characteristic stretching vibrations of TA-dPGS $\left(1224 \mathrm{~cm}^{-1}\right)$ and $\mathrm{mPEG}_{1000}-\mathrm{SH}$ $\left(1105 \mathrm{~cm}^{-1}\right)$ for seven different mass-fractions of TA-dPGS between X(TA-dPGS) $=0-1$ via ATR-FTIR spectroscopy. For this, $1 \mu$ of the aqueous solutions were placed on the ATR-crystal and dried under air flow until the IR spectrum did not exhibit any further changes. AuNR-dispersions were measured in an analogous manner.

\subsection{2 $\mathrm{BaCl}_{2}$ induced agglomeration assay}

For a typical aggregation test, an aqueous dispersion of gold nanorods $(0.5 \mathrm{~mL}, 2.72 \mathrm{nM}, 1.6 \mathrm{OD})$ was incubated with either $\mathrm{BaCl}_{2}(0.5 \mathrm{~mL}, 66 \mathrm{mM}$, IS=200 mM) or $\mathrm{NaCl}(0.5 \mathrm{~mL}, 0.2 \mathrm{mM}$, IS=200 mM). Directly after addition of the salt, UV-VIS absorption spectra were recorded at $20 \mathrm{~s}$ time intervals.

\section{SPR-based competitive L-selectin binding assay}

Binding of AuNR-PEG and AuNR-dPGS to L-selectin was tested by inhibition of L-selectin binding to an artificial ligand via a competitive binding assay performed on a BIACore $\mathrm{X}$ device (GE Healthcare, Freiburg, Germany) $[9,10,33,34]$. Briefly, protein A coated gold nanoparticles $(15 \mathrm{~nm}$, Aurion, Wageningen, Netherlands) were loaded with L-selectin/Fc chimera (R\&D systems, Minneapolis, USA). The binding signal to the artificial ligand bound onto a streptavidin coated gold chip (GE Healthcare, Freiburg, Germany) was measured, and samples without gold nanorods were set to $100 \%$. In turn, binding signals of the L-selectin loaded gold nanoparticles preincubated with different concentrations of either AuNR-PEG or AuNR-dPGS were taken, yielding the respective dose-dependent curve.

\subsubsection{Parallel plate flow chamber assay}

The human leukemia cell line K562 stably transfected with L-selectin was used to study ligand binding in cell rolling assays. Cells were resuspended in Hanks' balanced salt solution (PAA, Pasching, Austria) to a final concentration of $1 \times 10^{6} \mathrm{cells} / \mathrm{ml}$ and transferred into a syringe. A parallel plate flow chamber ( $\mu$-slide VI, ibidi GmbH, Germany) was incubated with $30 \mu \mathrm{g} / \mathrm{ml}$ of the L-selectin ligand
PSGL-1-Fc (R\&D Systems, Wiesbaden, Germany) for $2 \mathrm{~h}$ at room temperature in order to coat the surface. Subsequently, the surface was blocked with bovine serum albumin $(2 \mathrm{mg} / \mathrm{ml})$ for $30 \mathrm{~min}$. The flow channel was connected to a high precision perfusion pump KDS 101 (KD Scientific, Holliston, MA, USA) and the assays were performed at a constant shear stress of $1 \mathrm{dyn} / \mathrm{cm}^{2}$. An inverted microscope IM (Carl Zeiss AG, Oberkochen, Germany) that had been equipped with a digital camera AxioCam MRc (Carl Zeiss AG, Jena, Germany) was used to visualize the cell rolling. To monitor inhibition of receptor-ligand interaction, cells were respectively preincubated for 10 min at $37^{\circ} \mathrm{C}$ with AuNR-dPGS or AuNR-PEG at given concentrations. Movies were taken at 100x magnification for a $1 \mathrm{~min}$ period and the number of rolling cells (flux) was determined by counting.

\subsubsection{Cytotoxicity assay}

Cytotoxicity of AuNR-CTAB, AuNR-PEG and AuNR-dPGS was analyzed in real time by the impedance based xCELLigence System (RTCA device, Roche Applied Science, Mannheim, Germany). Therefore a 96 well e-plate (Roche Applied Science, Mannheim, Germany) was precoated with collagen and filled with $50 \mu \mathrm{l}$ Epithelia-Cell-Growth-Medium + supplement mix (Promocell, Germany) $+1 \%$ penicillin/streptomycin (PAA, Germany) per well to take the blank values. After that, HUVECs were cultured (2500 cells/well) in corresponding wells yielding 100 $\mu \mathrm{l}$ volume per well. The cells were allowed to settle down for $30 \mathrm{~min}$ at RT before incubation at $37^{\circ} \mathrm{C}$ and $5 \% \mathrm{CO}_{2}$ for $24 \mathrm{~h}$. Proliferation was monitored by increasing impedance automatically by the RTCA.

Samples were added to the cells, yielding a final volume of $150 \mu \mathrm{l}$ and impedance was analyzed for another 4 days at $37^{\circ} \mathrm{C}$ and $5 \% \mathrm{CO}_{2}$.

Data collected during the experiment were analyzed with RTCA Software 1.2.1.1002 (Roche Applied Science).

\subsection{MSOT imaging}

\subsection{Animal model}

All animal experiments were performed with the approval of the Government of Upper Bavaria (Reference number 55.2-1-54-2532-179-11). We used a collagen induced arthritis CIA mouse model, which is widely used as an arthritis model, because of its strong similarity to human rheumatoid arthritis [31]. For the induction of the arthritis, Complete Freund's Adjuvant CFA, which consists of mineral oil and heat-killed Mycobacterium tuberculosis at a concentration of $1 \mathrm{mg} / \mathrm{ml}$, and a phosphate buffered saline (PBS) solution containing bovine type II collagen at a concentration of $1 \mathrm{mg} / \mathrm{ml}$ were mixed together in 
equal parts. $20 \mu \mathrm{l}$ of this compound were injected between the left knee and ankle. Within approximately 25-30 days after the injection, most animals developed moderate to advanced arthritis. All animals were examined daily for their general state of health and specifically for arthritis associated symptoms. Swelling, redness, and lameness of the arthritic leg were graded by an experienced veterinarian in a scale from 0 to 3 , where 0 stands for no, 1 for mild, 2 for moderate, and 3 for severe development of each symptom. For this study, 6 animals with arthritis were used (4 for AuNR-dPGS, 2 for AuNR-PEG).

\subsubsection{Optoacoustic imaging procedure and processing}

All MSOT measurements were performed in a custom-built real time optoacoustic imaging system adapted from previously described equipment [35]. Briefly, optical excitation was provided by a Q-switched Nd:YAG laser with a pulse duration of around $10 \mathrm{~ns}$ and a repetition rate of $10 \mathrm{~Hz}$ and a tunable range of $680-980 \mathrm{~nm}$. Homogeneous delivery of light to the sample was achieved using a fiber bundle split into 10 output arms. A cylindrically focused 256-element transducer array with a central frequency of $5 \mathrm{MHz}$ was used to detect and record the emitted ultrasound waves, allowing acquisition of transverse plane images. A moving stage enabled the imaging of different planes by the static illumination and detection devices. Measurements were performed in a temperature controlled water bath $\left(34^{\circ} \mathrm{C}\right)$ for acoustic coupling and a thin clear polyethylene membrane attached to the sample holder was used to keep the samples dry.

\subsubsection{In vitro imaging}

For in vitro imaging, cylindrical phantoms of $2 \mathrm{~cm}$ diameter were prepared using a gel made from distilled water containing Agar (Sigma-Aldrich, St. Louis, MO, USA) for jellification $(1.3 \% \mathrm{w} / \mathrm{w})$ and an intralipid 20\% emulsion (Sigma-Aldrich, St. Louis, $\mathrm{MO}$, USA) for light diffusion $(6 \% \mathrm{v} / \mathrm{v})$, which resulted in a gel with a reduced scattering coefficient of $\mu^{\prime} \mathrm{\approx}$ $10 \mathrm{~cm}^{-1}$. A cylindrical inclusion containing various concentrations of the sample of approximately $3 \mathrm{~mm}$ diameter was put approximately in the middle of the phantom, along with a tube containing classical black ink with an optical density of around 0.2 for intensity measurement references. Imaging was performed using 20 averages per illumination wavelength, from 680 to $900 \mathrm{~nm}$ in steps of $5 \mathrm{~nm}$.

\subsubsection{In vivo imaging}

Imaging of the animals was performed roughly from toes to hips using $0.5 \mathrm{~mm}$ steps in order to image the whole region possibly inflamed. After injection of $5 \mathrm{mg} / \mathrm{kg}$ of AuNR i.v. in the animals, a similar con- centration to what is classically used in the field, the lower half of the body was imaged with transversal slides at various time points, ranging from directly after injection to $24 \mathrm{~h}$ post-injection. Data acquisition was performed using 30 averages per illumination wavelengths, which were as followed: $700,730,760$, 800,830 , and $860 \mathrm{~nm}$. This resulted in an acquisition time of around 20 minutes.

Data processing was performed using the commercial suite proposed by iThera Medicals (Munich, Germany). Briefly, model based image reconstruction is performed on the raw data and followed by spectral unmixing, based on spectral fitting by the least square method.

For 3D-video reconstruction, 2D images from MSOT experiments were processed using Amira ${ }^{\circledR}$ (VGS, Burlington, MA, USA), using volume rendering of thresholded images stacks. The anatomical data, which came from $800 \mathrm{~nm}$ illumination experiments (grey), is displayed using 50\% transparency. The dPGS-AuNR volume (green) is displayed with $0 \%$ transparency.

\section{Results and Discussion}

\section{I. Gold nanorod functionalization: CTAB to dPGS}

Dendritic polyglycerolsulfate with a molecular weight of $10 \mathrm{kDa}$ was chosen as an active ligand for the functionalization of gold nanorods due to low-cost [36], confirmed targeting potential for inflammatory diseases [22-24], and strong electrosteric repulsion required for stabilizing large colloids under physiological conditions. Furthermore, the high molecular weight of the polymer favors a ligand exchange reaction due to entropic gain upon replacing smaller molecular weight ligands. The obtained polymer with a molecular weight of $\sim 10 \mathrm{kDa}$ presented approximately 70 functional groups at the periphery with a degree of amination and sulfation of $10 \%$ and $85 \%$, respectively. As a result, approximately seven amine groups are available for functionalization via amide coupling, while 60 sulfate groups form the predominant part of the polymer surface. TA-dPGS was synthesized by a simple amide coupling of $\mathrm{N}$-hydroxysuccinimide (NHS) activated thioctic acid (TA) which was prepared by a procedure established by Liu et al. [32], to the $10 \%$ amine functionalized dendritic polyglycerolsufate with a molecular mass of $10 \mathrm{kDa}$ as presented in Scheme 1A. After careful adjustment of the dimethylformamide/water solvent ratio, the reaction was performed with quantitative yields and resulted in approximately one equivalent of TA per polymer as confirmed by ${ }^{1} \mathrm{H}-\mathrm{NMR}$.

$\mathrm{CTAB}$ functionalized gold nanorods were syn- 
thesized by a slightly modified, one-step synthetic method established by Nikhil R. Jana [25], resulting in nearly monodisperse gold nanorods as confirmed by the transmission electron micrographs presented in the Additional file 1: Figure S1. In order to remove excess $C T A B$, the synthesized gold nanorods were centrifuged twice at $6500 \mathrm{rpm}$ at $35^{\circ} \mathrm{C}$ for $2 \mathrm{~h}$, the supernatant was removed, and the rods redispersed in water. Further purification would have resulted in aggregation due to deceeding of the critical $[\mathrm{CTAB}] /[\mathrm{AuNR}]$ ratio of $7.4 \cdot 10^{5}$ required for a stable AuNR-CTAB dispersions [37]. The subsequent ligand exchange reactions on the gold nanorods were performed by a two-step method that permitted the complete removal of free CTAB before the addition of the polyanion (Scheme 1B).
A

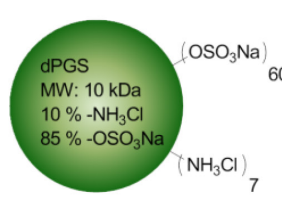

dPGS $10 \mathrm{kDa}$

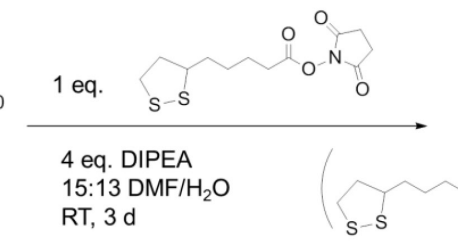

RT, 3 d

s-s
TA-dPGS $10 \mathrm{kDa}$

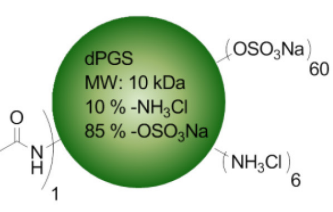

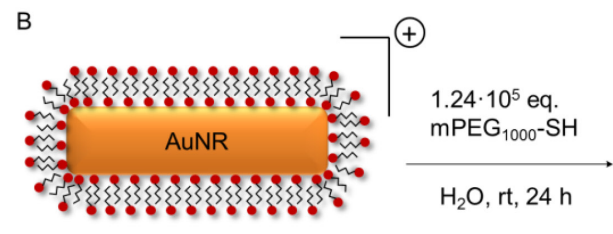

AuNR-CTAB

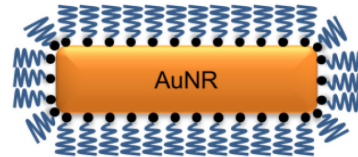

AuNR-mPEG ${ }_{1000}-\mathrm{SH}$
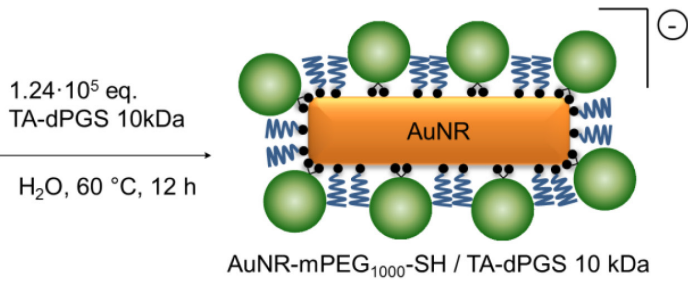

Scheme I. (A) Synthesis of thioctic acid functionalized dendritic polyglycerolsulfate via amide coupling. (B) Functionalization of CTAB double-layer coated gold nanorods with $\mathrm{mPEG}_{1000}$-SH followed by a partial replacement of $\mathrm{mPEG}_{1000}-\mathrm{SH}$ with TA-dPGS $10 \mathrm{kDa}$ via thermally induced ligand exchange reaction. Note: Elements in the scheme are not drawn to scale.

PEGylation was performed by incubating the AuNR-CTAB with $1.24 \cdot 10^{5}$ eq. of $\mathrm{mPEG}_{1000}-\mathrm{SH}$ as this ratio was identified to result in the highest possible degree of functionalization by a simple ligand exchange as the binding process is relatively slow [38]. The ligand exchange was confirmed macroscopically due to the perfect dispersability of the AuNRs in ethanol. As additional proof, an evenly spaced gold nanorod assembly with an interparticle diameter approximately resembling twice the length of an extended $\mathrm{mPEG}_{1000} \mathrm{SH}$ chain was visualized by transmission electron microscopy (TEM) (Additional file 1: Figure S1B). Attenuated total reflectance - fourier transform infrared spectroscopy (ATR-FTIR) measurements further confirmed the functionalization with $\mathrm{mPEG}_{1000}-\mathrm{SH}$ due to the appearance of the characteristic (C-O-C) stretching vibration at $1100 \mathrm{~cm}^{-1}$ (Figure 1).

Since residual CTAB and $\mathrm{mPEG}_{1000}-\mathrm{SH}$ would hinder the TA-dPGS ligand exchange on the nanorods, $\mathrm{mPEG}_{1000} \mathrm{SH}$ functionalized AuNRs were purified twice by centrifugation. Ligand exchange with TA-dPGS $10 \mathrm{kDa}$ was performed by incubation of the purified AuNR-PEG 1000 with $1.24 \cdot 10^{5}$ eq. of TA-dPGS $10 \mathrm{kDa}$ at $60^{\circ} \mathrm{C}$ over $12 \mathrm{~h}$. The elevated temperature of $60^{\circ} \mathrm{C}$ was applied in order to capitalize upon the entropic gain by exchanging the smaller AuNR-PEG ${ }_{1000}$ with TA-dPGS $10 \mathrm{kDa}$ and to accelerate the replacement of the mono-thiol with the more stable bivalent anchor moiety by breaking the thiol-gold coordinative bond. By estimating the surface area of a single gold nanorod and dividing it by an assumed footprint of one TA-dPGS $10 \mathrm{kDa}$ polymer of $1 \mathrm{~nm}^{2}, 1400$ TA-dPGS $10 \mathrm{kDa}$ molecules would be required for a complete surface coverage of one gold nanorod. Nevertheless, the 88 -fold amount, $1.24 \cdot 10^{5}$ eq., was chosen because the thiol-thiol exchange reaction follows Langmuir diffusion kinetics and the conversion was thus largely dependent on the incoming-outcoming ligand ratio [39]. Furthermore, we were able to show in this specific case that $1.24 \cdot 10^{4} \mathrm{eq}$. was not sufficient for a successful ligand exchange reaction. Neither was the functionalization with $1.24 \cdot 10^{5}$ eq. at room temperature successful, even after $72 \mathrm{~h}$ of incubation. The removal of unbound TA-dPGS $10 \mathrm{kDa}$ was achieved by five times centrifugation at $11,000 \mathrm{rpm}$ for $15 \mathrm{~min}$, disposal of the supernatant, and redispersion of the nanorods in water. The appearance of the strong stretching vibration characteristic for sulfate groups at $1250 \mathrm{~cm}^{-1}$ in the IR spectrum of purified AuNR-dPGS confirmed a successful functionalization with TA-dPGS $10 \mathrm{kDa}$ (Figure 1) [40]. 


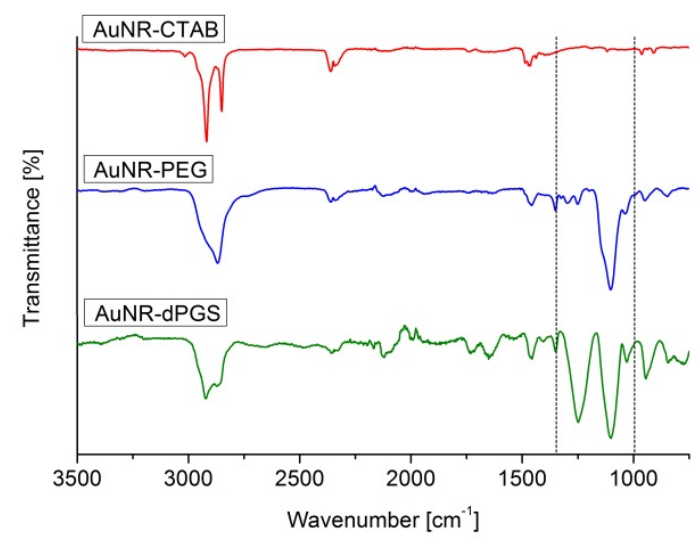

Figure I. ATR-FTIR spectra of the synthesized gold nanorods. The dotted lines mark the range of wavenumbers with characteristic changes.

Furthermore, in contrast to AuNR-PEG, AuNR-dPGS particles were no longer dispersible in ethanol and precipitated over time, which confirmed the successful functionalization on the macroscopic scale. The characteristic stretching vibrations of PEG and dPGS were further employed for determination of the dPGS to PEG mass ratio, which was identified to be $47.4+/-4.1 \%$ (Additional file 1: Figure S2, S3). Due to the 10-fold higher molecular mass of TA-dPGS, approximately 10 times more PEG molecules were present on the surface than TA-dPGS molecules. It is noteworthy that dPGS $10 \mathrm{kDa}$ has a hydrodynamic diameter of $6 \mathrm{~nm}$, which is much larger than the hydrodynamic diameter of $\mathrm{PEG}_{1000}$, beeing only $1.72 \mathrm{~nm}[10,41]$. The circular areas covered by one molecule of PEG 1000 and dPGS $10 \mathrm{kDa}$ were thus approximately $3 \mathrm{~nm}^{2}$ and $36 \mathrm{~nm}^{2}$, respectively. As there were 10 times more PEG molecules on the surface than dPGS $10 \mathrm{kDa}$ molecules, but with each covering only $1 / 12^{\text {th }}$ of the surface, half of the overall gold nanorod surface can be assumed to have been covered by TA-dPGS $10 \mathrm{kDa}$ sticking out of the shorter PEG layer. This maximum degree of functionalization that was achievable by the employed ligand exchange method appears to be reasonable as the highly charged, polyanionic dPGS molecules strongly repel each other. The formation of an evenly spaced TA-dPGS $10 \mathrm{kDa}$ lattice with mPEG $_{1000}-\mathrm{SH}$ in-between was thus favored. The addition of salt during the ligand exchange reaction could have increased the degree of dPGS functionalization due to the screening of charges [42], yet we did not encourage this. We have already shown that a higher degree of sulfate functionalization above $40 \%$ on a $6 \mathrm{~nm}$ dPGS only marginally increased the L-selectin binding affinity, indicating a cap for the multivalent enhancement of the binding affinity [10]. For a smaller dPGS of $3 \mathrm{kDa}$, higher degrees of functionalization were required to compensate the smaller size and thus number of binding sites in order to achieve maximum binding affinity. As the gold nanrods are at least one order of magnitude larger than the investigated dendritic polyglycerols, this cap should have been strongly exceeded even by $50 \%$ surface coverage of the gold nanorods with TA-dPGS. A further enhancement of the binding affinity of AuNR-dPGS by increasing the dPGS to PEG ratio is therefore unlikely. In this specific case, we even surmise that the mixed PEG/dPGS layer can beneficial for in vivo targeting of inflammation, as the clustered sulfate groups on the protein resistant PEG layer mimic the physiological selectin ligand distribution on the surface of inflamed endothelia and leukocytes. Zeta potentials measured by dynamic light scattering (DLS) were furthermore employed for monitoring the functionalization steps. The PEGylation of AuNR-CTAB resulted in a decreased zeta potential from $63.0+/-2.9 \mathrm{mV}$ to $5.12+/$ $0.8 \mathrm{mV}$, clearly indicating a successful ligand exchange reaction. The slightly positive zeta potential of the AuNR-PEG is due to some residual CTAB on the nanorod surface which could not be removed by this simple exchange reaction. Just recently, the group of Alke Petri-Fink established a protocol for removing this small residual amount as well by a two-step place exchange [43]. Nevertheless, this residual amount of $\mathrm{CTAB}$ is negligible, as it does not contribute to the cytotoxicity of gold nanorods [44]. The interpretation of the zeta potential after TA-dPGS $10 \mathrm{kDa}$ ligand exchange is ambiguous, as TA-dPGS $10 \mathrm{kDa}$ itself has a zeta potential of approximately $-14 \mathrm{mV}$ [10]. The significantly lower zeta potential for gold nanorods functionalized with TA-dPGS of $-36.8+/-0.85 \mathrm{mV}$ after extensive washing, confirmed a high degree of TA-dPGS functionalization but not the absence of unbound TA-dPGS. The conservation of the optical properties of the gold nanorods was approved via normalized absorption spectra which resulted in a LSPR band of $782 \mathrm{~nm}$ for AuNR-dPGS, which was perfectly suited for in vivo imaging (Additional file 1: Figure S4). Size determination by DLS measurements further confirmed the required stability in buffer for biological testing, as no aggregation could be observed for the AuNR-PEG and AuNR-dPGS (Additional file 1: Figure S6).

\subsection{Removal of unbound ligands}

The removal of unbound TA-dPGS from the colloidal solution after purification is essential for an accurate determination of the AuNR-dPGS targeting potential. Unfortunately, zeta potential measurements only give cumulative information about the colloidal solution and thus prohibit identification of unbound ligands. Even though altering SPR bands due to 
plasmon-plasmon interactions upon reduction of the interparticle diameter by cross-linking has been reported in literature [6,45-47], this has not been used as an analytic tool to assess colloidal purity. We employed $\mathrm{BaCl}_{2}$ to induce agglomeration of sulfate functionalized gold nanorods, which confirmed a successful functionalization with dPGS when the plasmon resonance was altered. The nanorod dispersion was purified prior to $\mathrm{BaCl}_{2}$ incubation several times by centrifugation. After each purification cycle, a small sample of the redispersed colloidal dispersion was removed for the $\mathrm{BaCl}_{2}$ agglomeration assay. The respective samples were incubated with $\mathrm{BaCl}_{2}$ and their absorption monitored via time-resolved UV-VIS spectroscopy. Surprisingly, no change in plasmon resonance, and thus agglomeration, was observed for samples of unpurified AuNR-dPGS after incubation with $\mathrm{BaCl}_{2}$. There was also no agglomeration for samples of AuNR-dPGS after four purification cycles (Additional file 1: Figure S7). Only samples of five-fold purified AuNR-dPGS exhibited a dramatic change of their plasmon resonance seconds after the addition of $\mathrm{BaCl}_{2}$ (Figure 2).

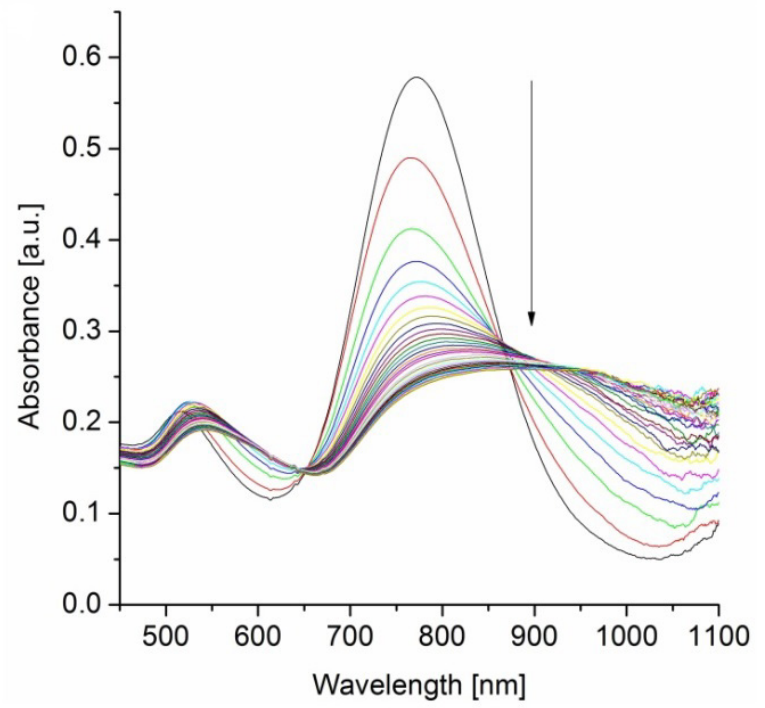

Figure 2. Time-resolved UV-VIS absorption spectra from 0-600 $\mathrm{s}$ in $20 \mathrm{~s}$ time steps after the addition of $\mathrm{BaCl}_{2}$ to five-fold purified AuNR-dPGS by centrifugation.

In order to exclude agglomeration effects due to high ionic strength, a control experiment was conducted in which samples of five-fold purified AuNRs-dPGS were incubated with $\mathrm{NaCl}$ at an ionic strength of $100 \mathrm{mM}$. The absence of plasmon coupling and thus agglomeration confirmed the stability of AuNR-dPGS at this ionic strength (Additional file 1: Figure S8). Furthermore, the UV-VIS absorption spectra of PEGylated AuNRs did not change after the addition of $\mathrm{BaCl}_{2}$, which proved the specific interaction of the polysulfate layer with $\mathrm{BaCl}_{2}$ (Additional file 1: Figure S9). We surmise that only in the case of highly purified AuNR-dPGS the interparticle distance of agglomerated gold nanorods were small enough for plasmon-plasmon interactions, as the electromagnetic field around the nanorods exponentially decays with distance. In the case of insufficiently purified gold nanorods, the approximately $5 \mathrm{~nm}$ large TA-dPGS ligands acted as an additional cross-linker between gold nanorods and thus separated the AuNR-dPGS to an interparticle distance too large for alterations of the plasmon resonance. In order to confirm the specific agglomeration of purified AuNR-dPGS by $\mathrm{BaCl}_{2}$, transmission electron micrographs were recorded as shown in Figure 3.

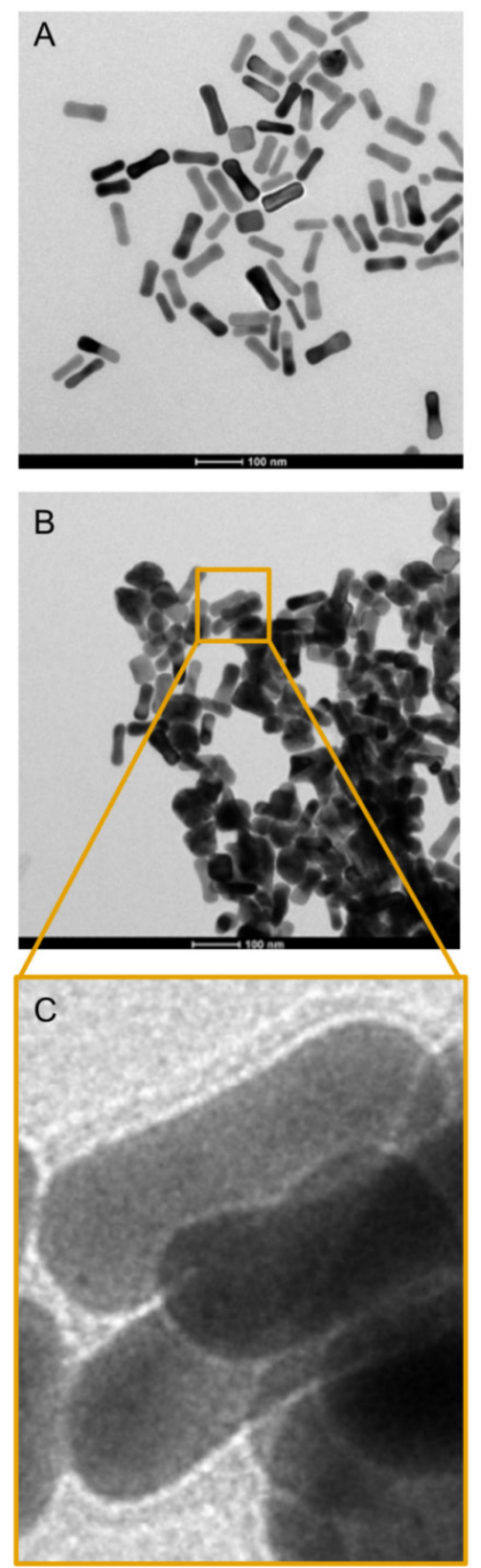

Figure 3. Transmission electron micrographs of purified AuNR-dPGS incubated with (A) $\mathrm{NaCl}$ and (B) $\mathrm{BaCl}_{2}$ at an ionic strength of $100 \mathrm{mM}$. (C) $A$ magnified detail from (B) depicts a dark seam around the nanorods indicating the $\mathrm{dPGS}$ corona. 
No agglomeration was observed for purified AuNR-dPGS incubated with $\mathrm{NaCl}$ (Figure 3A). On the contrary, incubation with $\mathrm{BaCl}_{2}$ induced strong agglomeration of AuNR-dPGS as depicted in Figure 3B, which confirms a solely $\mathrm{Ba}^{2+}$-sulfate induced agglomeration (for even larger agglomerates see Figure S11 in Additional file 1). Due to the high atomic mass of $\mathrm{Ba}, \mathrm{BaCl}_{2}$ acted as a positive staining agent and successfully visualized the dPGS corona as presented in Figure 3C. In the case of AuNR-PEG, incubation with $\mathrm{BaCl}_{2}$ neither resulted in aggregation nor staining of the nanorods, confirming the specific, positive staining of sulfates with $\mathrm{BaCl}_{2}$ (Additional file 1: Figure S12). The small interparticle distance between the AuNR-dPGS upon incubation with $\mathrm{BaCl}_{2}$ further explains the change in the SPR bands as plasmon coupling occurs. The assay was furthermore employed to prove the covalent binding of the TA-dPGS to the gold nanorod, as AuNR functionalized in an analogous manner with dPGS without thioctic acid moiety and did not show any signs of agglomeration after incubation with $\mathrm{BaCl}_{2}$ (Additional file 1: Figure S10).

\subsection{In vitro determination of AuNR-dPGS L-selectin and leukocyte binding}

In order to acquire quantitative information about the binding strength of AuNR-dPGS to L-selectin, a competitive binding assay based on surface plasmon resonance measurements (SPR) was performed $[9,10,33,34]$. In this assay, the binding of L-selectin coated $15 \mathrm{~nm}$ gold nanoparticles (AuNP) with a synthetic L-selectin ligand-modified sensor chip was measured as the positive control and normalized to $100 \%$ binding. Subsequently, the L-selectin coated AuNPs were preincubated with increasing concentrations of the respective gold nanorod sample and the relative binding was determined (Figure 4A). While AuNR-PEG did not show any inhibition of L-selectin ligand binding, AuNR-dPGS proved to be a potent inhibitor with an $\mathrm{IC}_{50}$ value of $135 \mathrm{pM}$, calculated on the basis of the nanorod concentration. Removal of the unbound TA-dPGS was essential for an accurate determination of the binding affinity, since soluble dPGS already exhibits an $\mathrm{IC}_{50}$ value of $8 \mathrm{nM}$ [10].

In order to test the efficiency of AuNR-dPGS as a L-selectin inhibitor under more physiological conditions, the nanorods were applied in a cellular based flow chamber assay (Figure 4B) [10,48]. Therefore, L-selectin transfected K562 cells were passed through a PSGL-1-coated flow channel under a constant shear stress of $1 \mathrm{dyn} / \mathrm{cm}^{2}$. The number of rolling cells (flux) of the untreated cells was set to $100 \%$. The flux of the treated cells (preincubated with AuNR-dPGS and AuNR-PEG) was calculated as a percentage flux of the control (representative movies are provided in the Supplementary Material). Figure 4B confirms a strong binding of AuNR-dPGS to the cells with an $\mathrm{IC}_{50}$ value of $9 \mathrm{nM}$. The nearly 10 -fold higher $\mathrm{IC}_{50}$ value compared to the SPR measurements is due to the much higher concentration of L-selectin presented on the cells in comparison to the gold nanoparticles in the SPR assay.

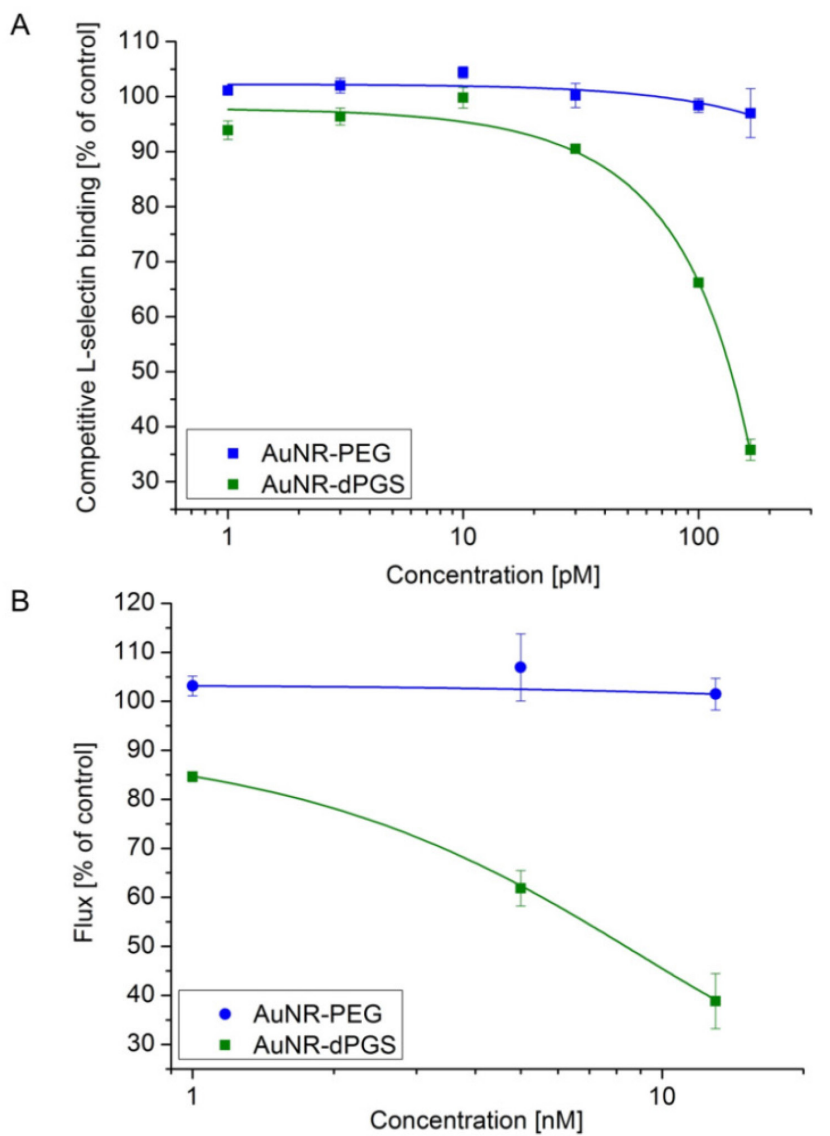

Figure 4. (A) Competitive L-selectin binding with different concentrations of gold nanorods. (B) Inhibition of leukocyte binding to immobilized ligands in a modified flow chamber by AuNR-dPGS. Data correspond to mean + /- SEM.

\subsection{In vitro cytotoxicity of AuNRs}

The cytotoxicity of the synthesized gold nanorods on human umbilical vein endothelial cells (HUVECs) was evaluated via a real time cell analyzer (RTCA) based on impedance measurements (Additional file 1: Figure S13). For AuNR-CTAB, particle concentrations $>0.1 \mathrm{nM}$ already resulted in complete death of the HUVECs after 1 day of incubation. The high cytotoxicity is in agreement with the results of Wyatt and coworkers, who have previously shown that AuNR-CTAB has a strong cytotoxic effect on HT-29 cells in concentrations of $\geq 0.2 \mathrm{nM}$ after 4 days of exposure [49]. AuNR-CTAB`s high cytotoxicity was ascribed to the unbound $\mathrm{CTAB}$ in solution required to 
stabilize the gold nanorods and not to particle bound CTAB. PEGylation and subsequent purification removed the CTAB and thus significantly lowered the cytotoxicity of AuNR-PEG by $>40$-fold, as even concentrations as high as $20 \mathrm{nM}$ did not show any cytotoxic effect on the proliferation of HUVECs. AuNR-dPGS was not cytotoxic at concentrations $<5 \mathrm{nM}$. Possible changes in the cytotoxicity due to aggregation can be excluded [50], as the stability of AuNR-PEG and AuNR-dPGS in PBS was confirmed by DLS measurements (Additional file 1: Figure S6). Van Leeuwen and coworkers investigated the cytotoxicity of gold nanorods functionalized with polystyrene sulfonate by a LBL approach (AuNR-PSS) [44]. For the investigated cell lines, i.e., SKBR3, CHO, C2C12, HL60, 50\% cell death was observed in all cases after 1 day of incubation with concentrations $\leq 0.05 \mathrm{nM}$. We surmise that the significantly lower cytotoxicity of AuNR-dPGS was from the absence of complexed CTAB. As the LBL functionalization of gold nanorods prohibits the complete removal of $\mathrm{CTAB}$ from the solution before the addition of the polyanion, large amounts of $\mathrm{CTAB}$ are complexed within the polyanionic layer. The thermally induced ligand exchange of PEG permitted the removal of unbound $\mathrm{CTAB}$ before addition of the polyanion and thus significantly lowers the resulting cytotoxicity.

\subsection{AuNR-dPGS as MSOT contrast agents}

In order to compare the influence of the grafting dPGS onto the surface of AuNR with the more commonly used PEG coating, we synthesized both types of surface functionalization and their properties investigated with an optoacoustic imaging system. Figure 5 shows spectra and sensitivity assays in MSOT. In Figure 5A, it can be seen that the light absorbance remained very similar even though surface functionalization changed. Using a dPGS layer did not induce aggregation as this would have changed the absorbance spectra by inducing a significant bathochromic shift. Reconstitution of the light absorbance from the optoacoustic signal perfectly fitted the light absorbance of the compound measured in the spectrometer. By looking at the different concentrations of gold nanorods, expressed by the optical density (OD), we investigated the concentration dependent optoacoustic signal generation in phantoms. Figure 5B shows that the overall optoacoustic spectra shape remained the same at different concentrations, enabling identification of the compound at concentrations as low as 0.05 OD. Figure 5C displays optoacoustic signals as a function of concentration and indicates a linear correlation between the two, with a coefficient of determination of more than 0.92 . With the confirmation that the AuNR-dPGS can be identified in a quantita- tive manner in the optoacoustic system, the stage is set for in vivo experiments.
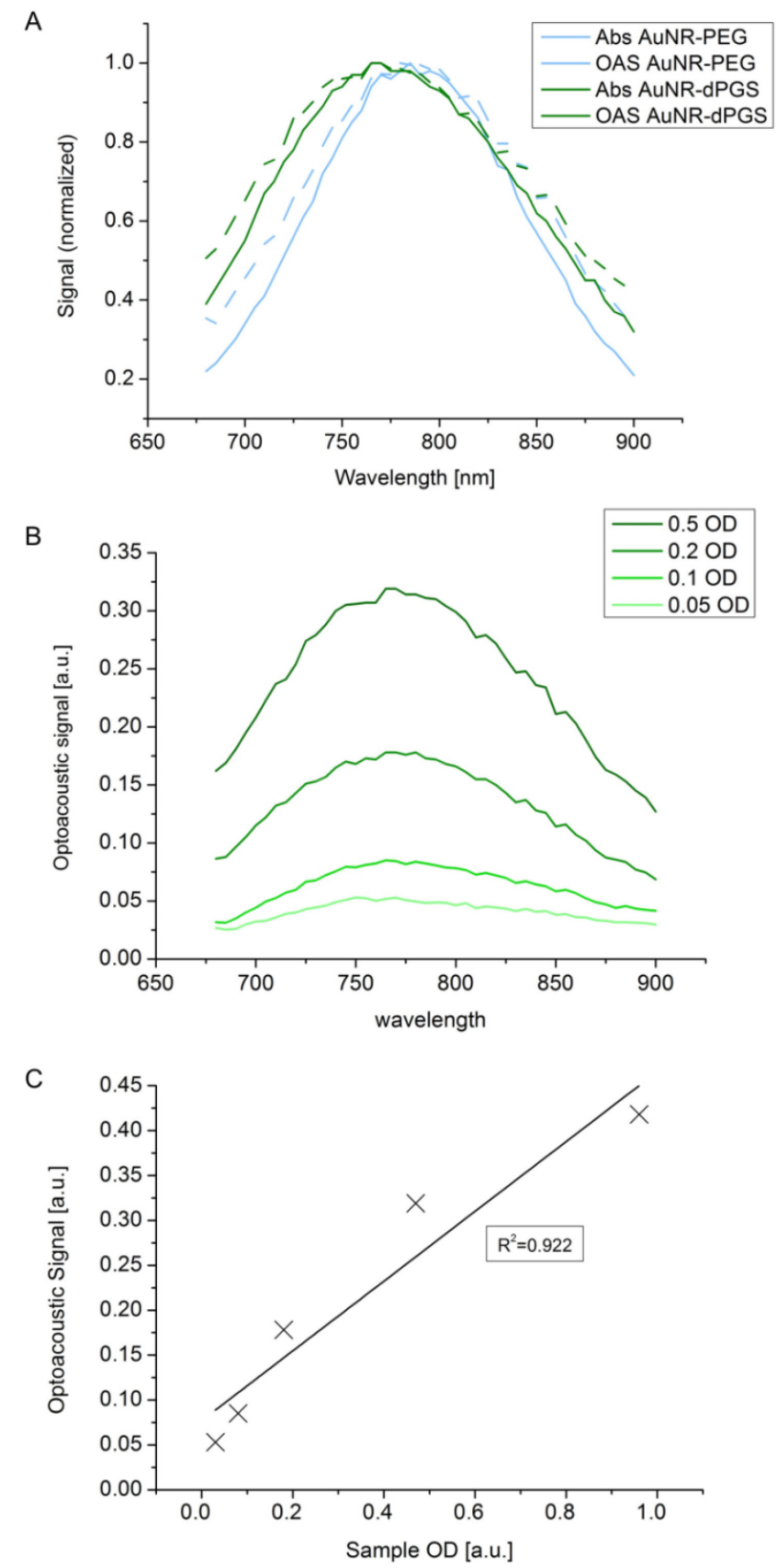

Figure 5. (A) Phantom study of AuNR-dPGS as optoacoustic imaging contrast agents. Optoacoustic signal (OAS) and light absorbance (Abs) comparison of gold nanorods functionalized with either PEG or dPGS. (B) Wavelength-dependent influence of the concentration of AuNR-dPGS on the optoacoustic signal shape at different optical densities. (C) Intensity of the optoacoustic signal relative to the concentration of the AuNR-dPGS sample.

Further investigations to estimate the potency of the AuNR-dPGS in vivo were performed with a well-established murine arthritis model that selectively triggered rheumatoid arthritis in the left injected limb. Figure 6 compares transverse images of two representative sample animals that exhibited 
moderate arthritis (stage 2 using clinical criteria) in their left ankle $4 \mathrm{~h}$ after intravenous injection with gold nanorods bearing either PEG or dPGS. (3D models of the lower part of the animals injected with AuNR-dPGS and AuNR-PEG can be found in supplementary movies). From the observed images acquired at the maximum absorbance wavelength of the gold nanorods $(800 \mathrm{~nm}$, Figure 6A and 6D), no molecular information can be extracted because they depict both nanoparticle and blood signal. Spectral unmixing depicted in Figure $6 \mathrm{~B}$ and $6 \mathrm{E}$ was done to identify and determine the exact location and accumulation of the nanoparticles in the joint. The same processing technique can be applied to blood, in the form of oxygenated hemoglobin, to yield a vasculature map of the region of interest and is presented in panels Figure 6C and 6F.

The colocalizing signals in Figure $6 \mathrm{~B}$ and $6 \mathrm{C}$ nicely show that AuNR-PEG did not seem to accumulate in either articulation as expected, and preferentially stayed in the blood flow as both signals colocalized nicely. This is justified by the long circulation times typically exhibited by PEG coated particles in vivo. In that case, the signals provided by the gold on both sides were equivalent with a difference of a mean signal value of less than $10 \%(n=2)$. Because of its inflammation targeting characteristics, AuNR-dPGS featured a significantly higher signal in the left (arthritic) ankle of the animal. When measuring the mean signal values shown in Figure 6, the left ankle exhibited 1.7 times more signal than the right side control (4.59 to 2.73 a.u., respectively). On average, the arthritic leg of animals injected with AuNR-dPGS presented $1.7 \pm 0.3$ times more signal than their healthy leg $(n=4)$. As AuNR-dPGS binds to the vascularly presented P-selectin as an inflammation specific target, a staining of the respective blood vessel section was observed. Furthermore, the AuNR-dPGS signal clearly diffused from the blood vessels of the arthritic ankle into the inflamed tissue. This indicates that the enhanced permeability of the vasculature per se is not sufficient to allow for extravasation of the nanorods, but that the active targeting, achieved here by the dPGS functionalization, appears to be necessary [51]. In a recent study, we described the tissue accumulation of dPGS in a comparable arthritic mouse model and found a colocalization with the infiltrate of inflammatory cells [22]. Therefore, it is possible that AuNR-dPGS recognized and bound to leukocytes and co-extravasate to the inflammatory tissue [52]. In addition, specific binding of AuNR-dPGS to inflammation relevant cytokines is conceivable. In the case of dPGS functionalization, this active targeting has the advantage of facilitating retention of the contrast agent, something which is impossible with untargeted AuNR-PEG. In imaging terms, this translates into an accumulation of nanoparticles and a noticeable increase in contrast, which enables diagnosis of this inflammatory disease through imaging and a direct distinction between healthy and arthritic joints. Finally, it is our hope that AuNR-dPGS turns out to act as a theragnostic agent that in addition to its imaging property also dampends inflammation due to its anti-complement activity [9].

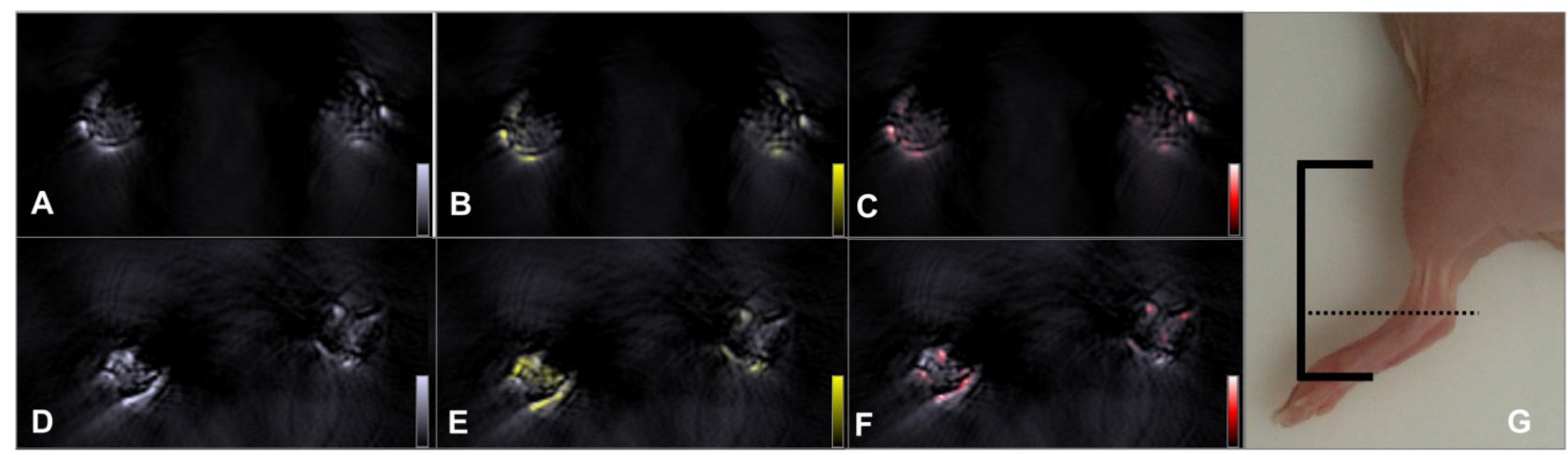

Figure 6. Accumulation of gold nanorods in the ankle of an arthritic mouse. Top row: anatomic optoacoustic images acquired at (A) $800 \mathrm{~nm}$ illumination wavelength, (B) overlayed with the signal coming from AuNR-PEG (yellow scale) or (C) with oxygenated hemoglobin signal (red scale). Bottom row: anatomic optoacoustic image acquired at (D) $800 \mathrm{~nm}$ illumination wavelength, (E) overlayed with the signal coming from AuNR-dPGS (yellow) or (F) with oxygenated hemoglobin signal (red scale). (G) Photograph of a mouse leg indicating the scanning region (solid lines) with the corresponding imaging plane displayed in the left panels (dotted line).

\section{Conclusions}

Dendritic polyglycerolsulfate functionalized gold nanorods were shown to be a promising candidate as contrast agent for the imaging of inflammatory diseases, i.e., rheumatoid arthritis, via MSOT.
Gold nanorods were functionalized with dPGS by a thermally induced exchange of PEG-thiol ligands on the nanorod surface. This novel ligand exchange requires no additional solvents or challenging reaction conditions and offers a universal way for the covalent 
functionalization of gold nanorods with hydrophilic ligands. The complete removal of CTAB before addition of polyanions significantly lowers the cytotoxicity of the resulting gold nanorods in comparison to other polysulfated gold nanorods reported in literature. Furthermore, we were able to show that the changes of SPR bands by plasmon coupling upon agglomeration can be utilized for the confirmation of a quantitative removal of unbound ligands with high sensitivity. The inflammation targeting potential of the AuNR-dPGS, which was evaluated in vitro by SPR in a competitive L-selectin binding assay, revealed a striking $\mathrm{IC}_{50}$ value of $135 \mathrm{pM}$. Functional testing under more physiological conditions in a flow chamber further confirmed inhibition of L-selectin mediated leukocyte adhesion with an $\mathrm{IC}_{50}$ below $10 \mathrm{nM}$. Further investigation on the efficiency of the dPGS coated colloid was performed with an in vivo imaging technique that allowed visualization, identification, and mapping of the gold nanorods without any chemical alteration. MSOT studies in a rheumatoid arthritis model were not only able to visualize and identify the signal of the nanorods, but were also able to highlight the better targeting potential of the functional polymer coating than classical AuNR-PEG, thereby inducing an significant contrast difference between healthy and arthritic joints. By demonstrating the synthesis, in vitro properties, and in vivo imaging possibilities offered by AuNR-dPGS, we show a promising tool for enabling diagnosis of inflammatory diseases through volumetric molecular imaging using the example of rheumatoid arthritis. Even though the biodistribution and in vivo toxicity remain to be investigated, the combination of the readily available gold nanorods as an imaging modality and the low-cost of dPGS for targeting paves the way for further research on other inflammatory diseases. Particularly when photothermal therapy can be applied using the gold core of the nanoparticle for cancer for example, the AuNRs-dPGS has great theranostic potential. Furthermore, as the residual amine groups on the TA-dPGS can be further functionalized with additional drugs or imaging modalities, AuNR-dPGS are perfectly suited as a platform for future research in multimodal imaging and theranostics.

\section{Supplementary Material}

Additional File 1:

Figure S1-S13.

http://www.thno.org/v04p0629s1.pdf

Additional File 2:

Leukocyte binding flow chamber assay.

http://www.thno.org/v04p0629s2.avi

Additional File 3:

MSOT imaging of arthritic mice - the left ankles are inflamed.

http://www.thno.org/v04p0629s3.avi

\section{Acknowledgements}

We acknowledge Luisa Russell, the Core Facility BioSupraMol (www.biosupramol.de) and the Focus Area Nanoscale of the Freie Universität Berlin (www.nanoscale.fu-berlin.de) for their practical and technical support. The Deutsche Forschungsgemeinschaft (SFB 765), the European Research Council Advanced Grant "Next Generation in-vivo imaging platform for post-genome biology and medicine (MSOT)" (Grant Agreement Number 233161) and the BMBF (Federal Ministry of Education and Research, Germany) excellence cluster $\mathrm{m}^{4}$ ("Individualized Medicine") (Y.K., M.K., R.M.) are acknowledged for funding.

\section{Competing Interests}

The authors have declared that no competing interest exists.

\section{References}

1. Ntziachristos V, Razansky D. Molecular imaging by means of multispectral optoacoustic tomography (MSOT). Chem Rev. 2010; 110: 2783-94.

2. Ntziachristos V. Going deeper than microscopy: the optical imaging frontier in biology. Nat Methods. 2010; 7: 603-14.

3. Manohar S. Gold nanorods as molecular contrast agents in photoacoustic imaging: the promises and the caveats. Contrast Media Mol Imaging. 2011; 6: 389-400.

4. Bao C, Beziere N, del Pino P, et al. Gold nanoprisms as optoacoustic signal nanoamplifiers for in vivo bioimaging of gastrointestinal cancers. Small. 2013; 9: 68-74

5. Tomography O, Herzog E, Taruttis A, et al. Optical imaging of cancer heterogeneity with Multispectral. Radiology. 2012; 263: 461-8.

6. Huang X, Neretina S, El-Sayed M. Gold nanorods: from synthesis and properties to biological and biomedical applications. Adv Mater. 2009; 19122: 4880-910.

7. Zhang Z, Wang J, Chen C. Gold nanorods based platforms for light-mediated theranostics. Theranostics. 2013; 3: 223-38.

8. Alkilany AM, Thompson LB, Boulos SP, et al. Gold nanorods: Their potential for photothermal therapeutics and drug delivery, tempered by the complexity of their biological interactions. Adv Drug Deliv Rev. 2012; 64: 190-9.

9. Dernedde J, Rausch A, Weinhart M, et al. Dendritic polyglycerol sulfates as multivalent inhibitors of inflammation. PNAS. 2010; 107: 19679-84.

10. Weinhart M, Gröger D, Enders S, et al. The role of dimension in multivalent binding events: structure-activity relationship of dendritic polyglycerol sulfate binding to L-selectin in correlation with size and surface charge density. Macromol Biosci. 2011; 11: 1088-98.

11. Ley $\mathrm{K}$. The role of selectins in inflammation and disease. Trends Mol Med. 2003; 9: 263-8.

12. Jung U, Ley K, Alerts E. Mice Lacking Two or All Three Selectins Demonstrate Overlapping and Distinct Functions for Each Selectin. J Immunol. 2014; 162: 6755-62.

13. Lowe JB. Glycosylation in the control of selectin counter-receptor structure and function. Immunol Rev. 2002; 186: 19-36.

14. Everts M, Kok RJ, Ásgeirsdóttir SA, et al. Selective Intracellular Delivery of Dexamethasone into Activated Endothelial Cells Using an E-Selectin-Directed Immunoconjugate. J Immunol. 2014; 168: 883-9.

15. Lindner JR, Song J, Christiansen J, et al. Ultrasound Assessment of Inflammation and Renal Tissue Injury With Microbubbles Targeted to P-Selectin. Circulation. 2001; 104: 2107-12.

16. Gompels LL, Madden $\mathrm{L}$, Lim NH, et al. In vivo fluorescence imaging of E-selectin: quantitative detection of endothelial activation in a mouse model of arthritis. Arthritis Rheum. 2011; 63: 107-17.

17. Sanders WJ, Katsumoto TR, Bertozzi CR, et al. L-Selectin-Carbohydrate Interactions : Relevant Modifications of the Lewis x. Biochemistry. 1996; 2960: 14862-7

18. Somers WS, Tang J, Shaw GD, et al. Insights into the Molecular Basis of Leukocyte Tethering and Rolling Revealed by Structures of P- and E-Selectin Bound to SLe X and PSGL-1. Cell. 2000; 103: 467-79. 
19. Koenig A, Jain R, Karin RV, et al. Selectin inhibition : synthesis and evaluation of novel sialylated, sulfated and fucosylated oligosaccharides, including the major capping group of GlyCAM-1. Glycobiology. 1997; 7: 79-93.

20. Woelke AL, Kuehne C, Meyer $\mathrm{T}$, et al. Understanding selectin counter-receptor binding from electrostatic energy computations and experimental binding studies. J Phys Chem B. 2013; 117: 16443-54.

21. Weinhart M, Gröger D, Enders S, et al. Synthesis of dendritic polyglycerol anions and their efficiency toward L-selectin inhibition. Biomacromolecules. 2011; 12: 2502-11.

22. Licha $K$, Welker $P$, Weinhart $M$, et al. Fluorescence imaging with multifunctional polyglycerol sulfates: novel polymeric near-IR probes targeting inflammation. Bioconjugate Chem. 2011; 22: 2453-60.

23. Taruttis A, Wildgruber M, Kosanke K, et al. Multispectral optoacoustic tomography of myocardial infarction. Photoacoustics. 2013; 1: 3-8.

24. Biffi S, Dal Monego S, Dullin C, et al. Dendritic polyglycerolsulfate near infrared fluorescent (NIRF) dye conjugate for non-invasively monitoring of inflammation in an allergic asthma mouse model. PLoS One. 2013; 8: e57150.

25. Jana NR. Gram-scale synthesis of soluble, near-monodisperse gold nanorods and other anisotropic nanoparticles. Small. 2005; 1: 875-82.

26. Gole A, Murphy C. Polyelectrolyte-coated gold nanorods: synthesis, characterization and immobilization. Chem Mater. 2005; 3: 1325-30.

27. Villiers MM De, Otto DP, Strydom SJ, et al. Introduction to nanocoatings produced by layer-by-layer ( LbL ) self-assembly. Adv Drug Deliv Rev. 2011; 63: 701-15.

28. Hu X, Gao X. Multilayer coating of gold nanorods for combined stability and biocompatibility. Phys Chem Chem Phys. 2011; 13: 10028-35.

29. Wijaya A, Hamad-Schifferli K. Ligand customization and DNA functionalization of gold nanorods via round-trip phase transfer ligand exchange. Langmuir. 2008; 24: 9966-9.

30. Tsai D-H, Shelton MP, DelRio FW, et al. Quantifying dithiothreitol displacement of functional ligands from gold nanoparticles. Anal Bioanal Chem. 2012; 404: 3015-23.

31. Brand DD, Latham K a, Rosloniec EF. Collagen-induced arthritis. Nat Protoc. 2007; 2: 1269-75.

32. Liu W, Howarth M, Greytak AB, et al. Compact Biocompatible Quantum Dots Functionalized for Cellular Imaging. JACS. 2008; 130(4):1274-84.

33. Papp I, Dernedde J, Enders S, et al. Modular synthesis of multivalent glycoarchitectures and their unique selectin binding behavior. chem commun. 2008; 44:5851-3

34. Enders S, Bernhard G, Zakrzewicz A, et al. Inhibition of L-selectin binding by polyacrylamide-based conjugates under defined flow conditions. BBA. 2007; 1770: 1441-9.

35. Rosenthal A, Razansky D, Ntziachristos V. Quantitative optoacoustic signal extraction using sparse signal representation. Opt Express. 2009; 28: 1997-2006.

36. Gröger D, Paulus F, Licha K, et al. Synthesis and biological evaluation of radio and dye labeled amino functionalized dendritic polyglycerol sulfates as multivalent anti-inflammatory compounds. Bioconjugate Chem. 2013; 24: 1507-14.

37. Rostro-Kohanloo BC, Bickford LR, Payne CM, et al. The stabilization and targeting of surfactant-synthesized gold nanorods. Nanotechnology. 2009; 20: 434005.

38. Boca SC, Astilean S. Detoxification of gold nanorods by conjugation with thiolated poly(ethylene glycol) and their assessment as SERS-active carriers of Raman tags. Nanotechnology. 2010; 21: 235601.

39. Kassam A, Bremner G, Clark B, et al. Place exchange reactions of alkyl thiols on gold nanoparticles. JACS. 2006; 128: 3476-7.

40. Saad P, Flach C. Infrared spectroscopic studies of sodium dodecyl sulphate permeation and interaction with stratum corneum lipids in skin. Int J Cosmet Sci. 2012; 34(1) :36-43.

41. Verdon J, Falge M, Maier E, et al. Detergent-like activity and alpha-helical structure of warnericin RK, an anti-Legionella peptide. Biophys J. 2009; 97: 1933-40.

42. Hurst SJ, Lytton-jean AKR, Mirkin CA. Maximizing DNA Loading on a Range of Gold Nanoparticle Sizes. Anal Chem. 2006; 78: 8313-8.

43. Kinnear C, Dietsch H, Clift MJD, et al. Gold nanorods: controlling their surface chemistry and complete detoxification by a two-step place exchange. Angew Chem Int Ed. 2013; 52: 1934-8

44. Rayavarapu RG, Petersen W, Hartsuiker L, et al. In vitro toxicity studies of polymer-coated gold nanorods. Nanotechnology. 2010; 21: 145101.

45. Yang YI, Choi I, Hong S, et al. Selective Aggregation of Polyanion-Coated Gold Nanorods Induced by Divalent Metal Ions in an Aqueous Solution. J Nanosci Nanotechnol. 2010; 10: 3538-42.

46. Jain PK, Eustis S, El-Sayed M a. Plasmon coupling in nanorod assemblies: optical absorption, discrete dipole approximation simulation, and exciton-coupling model. J Phys Chem B. 2006; 110: 18243-53.

47. Basu S, Panigrahi S, Praharaj S, et al. Dipole-dipole plasmon interactions in self-assembly of gold organosol induced by glutathione. New J Chem. 2006; 30: 1333-9.

48. Buscher $\mathrm{K}$, Riese SB, Shakibaei $\mathrm{M}$, et al. The transmembrane domains of L-selectin and CD44 regulate receptor cell surface positioning and leukocyte adhesion under flow. J Biol Chem. 2010; 285: 13490-7.

49. Alkilany AM, Nagaria PK, Hexel CR, et al. Cellular uptake and cytotoxicity of gold nanorods: molecular origin of cytotoxicity and surface effects. Small. 2009; 5: 701-8.
50. Rivera-Gil P, Jimenez de Aberasturi D, Wulf V, et al. The challenge to relate the physicochemical properties of colloidal nanoparticles to their cytotoxicity. Acc Chem Res. 2013; 46: 743-9.

51. Williams TJ, Brain SD, Hellewell PG, et al. Alteration in microvascular permeability induced by products released during inflammation. Prog Clin Biol Res. 1988; 263: 55-69.

52. Vestweber D, Blanks JE. Mechanisms that regulate the function of the selectins and their ligands. Physiol Rev 1999; 79: 181-213. 